\title{
Does the metabolic syndrome help to select patients requiring high statin dose?
}

\author{
AJ Scheen \\ Department of Medicine, CHU Sart Tilman (B35), B-4000 Liège, Belgium
}

Statins offer remarkable protection against cardiovascular disease, with an acceptablesafetyprofile. ${ }^{1}$ People requiring statins are those at high risk of cardiovascular disease, irrespective of baseline lipid levels. ${ }^{2-4}$ This statement being accepted, two remaining key questions are: how to best stratify risk individually and how aggressive statin therapy should be according to baseline individual risk of cardiovascular disease. ${ }^{5}$

Controversies arose about the benefit-to-risk ratio of lowering cholesterol as much as possible in the absence of a high risk of cardiovascular disease, ${ }^{6}$ on the one hand, and about the meaning of the metabolic syndrome as an independent cardiovascular risk factor, on the other. ${ }^{7}$ In today's Lancet, Prakash Deedwania and colleagues ${ }^{8}$ add an interesting piece of information to this complex debate. In their post-hoc analysis of the Treating to New Targets (TNT) study, patients with coronary heart disease and metabolic syndrome benefited from aggressive atorvastatin therapy $(80 \mathrm{mg}$ instead of $10 \mathrm{mg}$ a day $)$, irrespective of the presence of diabetes. In patients receiving a low dose of atorvastatinthe incidence of major cardiovascular events was higher in the presence of metabolic syndrome (113\%; if diabetes excluded, $99 \%)$ than in the subgroup without metabolic syndrome (8\%; $7.5 \%$ if diabetes excluded). But the incidence remained lower in patients with metabolic syndrome than that in the diabetic population (17.9\%). The number needed to treat to avoid a major cardiovascular event over a 5-year period with $80 \mathrm{mg}$ versus $10 \mathrm{mg}$ atorvastatin averaged 45 in the whole TNT population; ${ }^{9}$ it increased up to 167 in the subgroup without metabolic syndrome,${ }^{8}$ but decreased to 24 in patients with diabetes, ${ }^{10}$ and to 28 in those with metabolic syndrome (without diabetes) (figure). ${ }^{8}$ Thus focusing aggressive therapy on patients with metabolic syndrome or diabetes in a population with stable coronary heart disease allows the number needed to treat to be reduced substantially, which thus gains a significant improvement in the cost-effectiveness of high statin dose.

Patients with coronary heart disease have, de facto, a high cardiovascular disease risk, making their individual risk calculation unnecessary before starting statin therapy. ${ }^{2-5}$ The key question is not whether a statin should be started in these patients, but rather how aggressive the treatment should be. Although acute coronary syndrome might justify a high dose of statin, ${ }^{4,5}$ the risk/benefit of such aggressive approach remains an open question in patients with stable coronary heart disease. ${ }^{6}$ The TNT findings suggest that the presence of metabolic syndrome might help to select patients who will best benefit from an aggressive lipid-lowering therapy. In the CASS registry in North America, metabolic syndrome also conferred a higher risk of long-term death in patients with pre-existing coronary heart disease, and dysglycaemia seemed to be responsible for most of the associated risk. ${ }^{11}$ In TNT, univariate analysis of the individual characteristics of metabolic syndrome showed a significant, and almost similar, increased risk of major cardiovascular events with the presence of each component of metabolic syndrome. Interestingly, the risk of major cardiovascular events increased with the presence of each additional component of metabolic syndrome.

In patients without cardiovascular disease, the decision about starting a statin relies on the calculation of a global cardiovascular disease risk, taking into account many classic risk factors, but not some others that form part of the metabolic syndrome. ${ }^{2-4}$ However, in the INTERHEART study, abdominal obesity, a key feature of metabolic syndrome, accounted for $44 \%$ of the risk of a first myocardial infarction. ${ }^{12}$ Unfortunately, because waist circumference was not measured in TNT, the two most popular definitions of metabolic syndrome (National Cholesterol Education Program, International Diabetes Federation) could not be used in their strict sense for the analysis. More importantly, the value of metabolic syndrome compared with the Framingham equation for individual risk stratification has been challenged in primary prevention. ${ }^{13}$ In the San Antonio Heart Study, metabolic syndrome predicts less effectively the future development of cardiovascular disease than the established Framingham risk score, and does not enhance the performance of the Framingham score when combined with it. ${ }^{14}$

The results from TNT lend further support to implementing lower lipid goals than those currently suggested by guidelines. However, when treatment with high statin doses is considered, the selection of patients whose risk of major cardiovascular events is high enough to outweigh the potential risk of toxic drug-related effects is most 
important. The two post-hoc analyses of TNT in patients with diabetes and/or metabolic syndrome support the concept of the higher the risk, the greater the need to treat and to treat aggressively. This concept, already accepted in primary prevention, might be extended to secondary prevention, although such a suggestion requires further assessment.

\section{Figure: Major cardiovascular events in TNT trial}

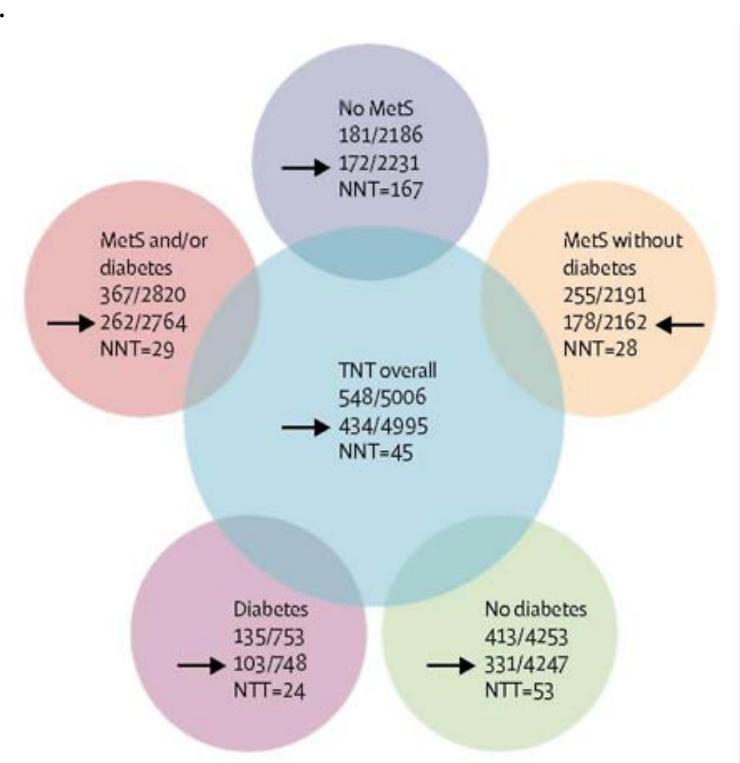

Number of major cardiovascular events per number of patients treated with atorvastatin $10 \mathrm{mg}$ (above each arrow) or atorvastatin 80 mg (atthe right of each arrow). NNT=number needed to treat, with high instead of low dose of atorvastatin to avoid major cardiovascular events over 5-year period in various subgroups. MetS=metabolic syndrome. The first row of numbers above each arrow corresponds to number of events per number of patients treated with atorvastatin $10 \mathrm{mg}$. Numbers at the right of each arrow corresponds to number of events per number of patients treated with atorvastatin

\section{References}

1 Baigent C, Keech A, Kearney PM, et al. Efficacy and safety of cholesterol-lowering treatment: prospective meta-analysis of data from 90056 participants in 14 randomised trials of statins. Lancet 2005; 366: 1267-78.

2 Grundy SM, CleemanJ, MerzCNB, for the Coordinating Committee of the National Cholesterol Education Program. Implications of recent clinical trials forthe National Cholesterol Education Program Adult Treatment Panel III Guidelines. Circulation 2004; 110: $227-39$.

3 DeBacker G, Ambrosioni E, Borch-Johnsen K, for the Third Joint Task Force of European and other Societies on cardiovascular disease prevention in clinical practice. European guidelines on cardiovascular disease prevention in clinical practice. Eur Heart J 2003; 24:1600-09.

4 British Cardiac Society, British Hypertension Society, Diabetes UK, Heart UK, Primary Care Cardiovascular Society, Stroke Association. JBS2: Joint British Societies' guidelines on prevention of cardiovascular disease in clinical practice. Heart 2005; 91 (suppl 5): v1-52.

5 Smith SC Jr, Allen J, Blair SN, for the AH A/ACC; National Heart, Lung, and Blood Institute. AH A/ACC guidelines for secondary prevention for patients with coronary and other atherosclerotic vascular disease: 2006 update: endorsed by the National Heart, Lung, and Blood Institute. Circulation 2006; 113: 2363-72.

6 Pedersen TR, Faergeman O, Kastelein JJP, forthe Increment Decrease in End PointsThrough Aggressive Lipid Lowering (IDEAL) Study Group. High-dose atorvastatin vs usual-dose simvastatin for secondary prevention after myocardial infarction. The I DEAL study: a randomized controlled trial. JAMA 2005;294:2437-45.

7 Kahn R, Buse J, Ferrannini E, Stern M. The metabolic syndrome: time for a critical appraisal: joint statement from the American Diabetes Association and the European Association forthe Study of Diabetes. Diabetologia 2005; 48:1684-99.

8 Deedwania P, Barter Ph, Carmena R, for the Treating to New targets Investigators.Reduction of low-density lipoprotein cholesterol in patients with coronary heart disease and metabolic syndrome: analysis of the Treating to New Targets study. Lancet 2006; published online Sept 5. DOI:10.1016/S0140-6736(06)69292-1.

9 LaRosa JC, Grundy SM, Waters DD, et al. Intensive lipid lowering with atorvastatin in patients with stable coronary disease. $N$ Engl $J$ Med 2005; 352: 1425-35. 
Published in: Lancet (2006), vol. 368, iss. 9539, pp. 893-4

Status: Postprint (Author's version)

10 Shepherd J, Barter P, Carmena R, fortheTreating to New Targets Investigators. Effect of lowering LDL cholesterol substantially below currently recommended levels in patients with coronary heart disease and diabetes: theTreating to New Targets (TNT) study. Diabetes Care 2006; $29: 1220-26$

11 Nigam A, Bourassa MG, Fortier A, Guertin M-C,Tardiff J-C The metabolic syndrome and its components and the long-term risk of death in patients with coronary heart disease. Am Heart J 2006; 151: 514-21.

12 Yusuf S, Hawken S, Ovnpvo S, on behalf of the INTERHEART Study Investigators. Obesity and the risk of myocardial infarction in 7000 participants from 52 countries: case-control study. Lancet 2005; 366: 1640-49.

13 Wilson PW. Estimating cardiovascular disease risk and the metabolic syndrome: a Framingham view. Endocrinol Metab Clin North Am 2004; 33: 467-81.

14 Stern MP, Williams K, Gonzalez-Villalpando C, Hunt KJ, Haffner SM. Does the metabolic syndrome improve identification of individuals at risk of type 2 diabetes and/or cardiovascular disease? Diabetes Care 2004; 27: 2676-81. 